\title{
The Impact of Bedside Interdisciplinary Rounds on Length of Stay and Complications
}

\author{
Andrew S. Dunn, MD, MPH*, Maria Reyna, MD¹, Brian Radbill, MD², Michael Parides, PhD, MS, MPhil ${ }^{3}$, \\ Claudia Colgan 4 , Tobi Osio ${ }^{5}$, Ari Benson, $\mathrm{MD}^{6}$, Nicole Brown, $\mathrm{MD}^{6}$, Joy Cambe ${ }^{6}$, Margo Zwerling, $\mathrm{MPH}^{6}$, \\ Natalia Egorova, $\mathrm{PhD}, \mathrm{MPH}^{3}$, Harold Kaplan, $\mathrm{MD}^{3}$
}

${ }^{1}$ Division of Hospital Medicine, Mount Sinai Health System, New York, New York; ${ }^{2}$ Mount Sinai Hospital Queens, Queens, New York; ${ }^{3}$ Department of Population Health Science and Policy, Mount Sinai Medical Center, New York, New York; ${ }^{4}$ Mount Sinai Health System, New York, New York; ${ }^{5}$ Department of Nursing, Mount Sinai Health System, New York, New York; ${ }^{6}$ Department of Medicine, Mount Sinai Health System, New York, New York.

BACKGROUND: Communication among team members within hospitals is typically fragmented. Bedside interdisciplinary rounds (IDR) have the potential to improve communication and outcomes through enhanced structure and patient engagement.

OBJECTIVE: To decrease length of stay (LOS) and complications through the transformation of daily IDR to a bedside model.

DESIGN: Controlled trial.

SETTING: 2 geographic areas of a medical unit using a clinical microsystem structure.

PATIENTS: 2005 hospitalizations over a 12-month period.

INTERVENTIONS: A bedside model (mobile interdisciplinary care rounds [MICRO]) was developed. MICRO featured a defined structure, scripting, patient engagement, and a patient safety checklist.

MEASUREMENTS: The primary outcomes were clinical deterioration (composite of death, transfer to a higher level of care, or development of a hospital-acquired complication) and length of stay (LOS). Patient safety culture and perceptions of bedside interdisciplinary rounding were assessed pre- and postimplementation.

RESULTS: There was no difference in LOS (6.6 vs 7.0 days, $P=0.17$, for the MICRO and control groups, respectively) or clinical deterioration $(7.7 \%$ vs $9.3 \%, P=0.46)$. LOS was reduced for patients transferred to the study unit (10.4 vs 14.0 days, $P=0.02$, for the MICRO and control groups, respectively). Nurses and hospitalists gave significantly higher scores for patient safety climate and the efficiency of rounds after implementation of the MICRO model.

LIMITATIONS: The trial was performed at a single hospital.

CONCLUSIONS: Bedside IDR did not reduce overall LOS or clinical deterioration. Future studies should examine whether comprehensive transformation of medical units, including co-leadership, geographic cohorting of teams, and bedside interdisciplinary rounding, improves clinical outcomes compared to units without these features. Journal of Hospital Medicine 2017;12:137-142. (C) 2017 Society of Hospital Medicine
The care of hospitalized patients requires practitioners from multiple disciplines to assess and communicate the patient's status in a dynamic manner during hospitalization. Although optimal teamwork is needed for patient care to be delivered reliably and efficiently, care within hospitals is typically delivered in a fragmented manner. ${ }^{1}$ A bedside model for daily interdisciplinary rounds (IDR) has been proposed as a method to provide a structured process and engage all team members in a patient-centered, system-of-care delivery. ${ }^{2}$ Specific advantages of convening rounds in the presence of the patient include the ability to directly assess care (eg, presence of a potentially unnecessary urinary catheter), patient engagement in key aspects of their care and disposition, and an

\footnotetext{
*Address for correspondence and reprint requests: Andrew S. Dunn, MD, MPH, Chief, Division of Hospital Medicine, Mount Sinai Health System, 1468 Madison Ave, Box 1087, New York, NY 10029; Telephone: 212-241-2920; Fax: 212-289-6393; E-mail: andrew.dunn@mountsinai.org
}

Additional Supporting Information may be found in the online version of this article. Received: March 17, 2016; Revised: July 22, 2016; Accepted: July 31, 2016 2017 Society of Hospital Medicine DOI 10.12788/jhm.2695 increased opportunity for team members to develop a shared understanding of the patient's views and needs.

Implementing dramatic changes to the workflow of multiple disciplines will require rigorous evidence to support a concerted effort from leadership and buy-in from stakeholders at the front line of patient care. Despite the urgency for evidence, there has been little investigation of this strategy. A systematic review ${ }^{3}$ identified 30 studies published between 1998 and 2013 addressing interdisciplinary interventions on medical wards, none of which examined a bedside IDR model. In a study performed after the period assessed by the systematic review, Stein et $\mathrm{al}^{4}$ described the restructuring of a medical ward as an accountable care unit (ACU), which included a bedside model for rounds by the interdisciplinary team. The change was associated with decreased mortality and length of stay (LOS), although the study did not isolate the impact of rounds or use a concurrent control group and presented aggregate rather than patient-level outcomes. The lack of convincing data may be a reason bedside rounds are not widely employed by hospitals. To provide high-quality evidence, we performed a large, prospective controlled trial comparing a structured bedside model (mobile interdisciplinary care rounds [MICRO]) with standard rounds. 


\section{METHODS}

This study took place at the Mount Sinai Hospital, which is a 1171-bed tertiary care academic medical center in New York City, New York. A nonteaching unit offered the ability to use a prospective controlled design. Patients were assigned to the north and the south wings of the unit in a quasi-randomized manner, rather than based on diagnosis or acuity. We transformed IDR to a bedside model on the north side of the unit (MICRO group), while the south side of the unit continued using standard conference roombased IDR (control group). The north and south sides of the unit contain 17 and 14 beds, respectively. During the study period, nurses and hospitalists cared for patients on both sides of the study unit, although on any given day were assigned only patients on 1 side of the unit. The unit uses a clinical microsystem model, which has been defined as "a group of clinicians and staff working together with a shared clinical purpose to provide care for a population of patients," and has a defined set of characteristics associated with high performance. ${ }^{5,6}$ Our microsystem model has incorporated features as described by Stein's ACU model, ${ }^{4}$ including co-leadership by a hospitalist and a nurse manager, geographic assignment of patients to teams, and unit-level data reports. One hospitalist is assigned geographically to each area of the unit in a 2-to 4-week rotation. Coverage of the unit does not include house staff; patients are primarily assigned to hospitalists working with nurse practitioners. Patients were enrolled prospectively during their initial IDR by a research coordinator. Patient-level data and outcomes were collected prospectively by a research coordinator who attended IDR on the intervention and the control sides of the study unit daily.

\section{Inclusion Criteria}

All patients admitted to the medicine service on the study unit were eligible. Patients were greater than 18 years and admitted for an acute medical condition. Patients admitted to another unit and later transferred to the study unit were enrolled at the time of transfer. Patients could be included more than once if hospitalized on the study unit on more than 1 occasion. Most patients were covered by hospitalists, although patients covered by private physicians were included. Patients from other departments, including family medicine, are uncommonly admitted to the unit and were excluded. Patients were also excluded if they were admitted and discharged over the same weekend, because the MICRO rounds occur during weekdays and there was no opportunity to offer the intervention on Saturdays and Sundays.

\section{MICRO Intervention}

Interdisciplinary rounds occurred daily at 10:00 AM for the control group and at 10:30 AM for the MICRO group, and were attended by the hospitalist caring for the majority of patients on the unit, staff nurses, and the unit medical director, nurse manager, social worker, and case manager. Rounds on the control unit focused on the plan of care and disposi- tion but did not follow any set structure and were typically 25 to 30 minutes in duration.

The MICRO rounds occurred at the bedside and followed a structured script (Appendix 1) that was designed to limit discussion of each patient to 3 minutes or less, and included speaking roles for the hospitalist, nurse, and social worker. For private physicians, the nurse practitioner assigned to the patient performed the role of the hospitalist. Rounds were expected to be approximately 50 minutes in duration. Patients were further engaged by asking for their main goal for the day. A patient safety checklist was reviewed. Initially, this task was performed by the nurse manager, who did not verbalize the items unless a deficiency was noted. After 6 months' experience, this responsibility was given to the staff nurse, who reviewed the checklist verbally as part of the bedside script. Patients were seen daily, including those being discharged later that same day.

\section{Staff and Clinician Education}

We developed and implemented a curriculum based on a modified version of the Agency for Healthcare Research and Quality's TeamStepps ${ }^{\circledR}$ program to ensure that all team members were provided with the basic principles of communication within the healthcare setting. The curriculum consisted of interactive didactics on essential elements of teamwork, including team structure, communication, situation monitoring, and mutual support, as well as the purpose and structure of the MICRO model. The curriculum was delivered to nurses at 3 monthly staff meetings on the study unit and to hospitalists during 3 hospital medicine grand rounds over a 3 -month period. Nurses and physicians providing care on both geographic areas of the study unit received the education program because no group of practitioners was designated to only 1 geographic area.

\section{OUTCOMES}

\section{Primary and Secondary Outcomes}

The primary outcomes were clinical deterioration (CD) and length of stay. Clinical deterioration was a composite outcome defined a priori as death; escalation of care (ie, transfer to an intensive care unit, intermediate care unit, or teaching unit); or a hospital-acquired complication (ie, venous thromboembolism, fall, stage III-IV pressure ulcer, catheter-associated urinary tract infection, central-line associated bloodstream infection, or Clostridium difficile-associated diarrhea). The LOS was calculated as the mean LOS with outliers excluded (outliers defined as having a LOS 100 days or longer or 2.5 or more standard deviations from the expected LOS).

Process metrics on IDR, such as the duration of rounds, attendance by members of the interdisciplinary team, the percentage of patients discussed, or the effectiveness of communication, were not collected. We assessed patient satisfaction based on the Hospital Consumer Assessment of Healthcare Providers and Systems (HCAHPS) survey. 


\section{Patient Safety Culture Survey}

To assess the impact on the perceptions of patient safety, we administered the Agency for Healthcare Research and Quality (AHRQ) Hospital Survey on Patient Safety Culture to all staff and clinicians working on both sides of the study unit immediately before and 12 months after implementation of the MICRO model. Results are reported for the AHRQ dimensions that were most relevant to the MICRO intervention: "teamwork within units," "overall perceptions of safety," "communication," "openness," "overall patient safety grade," and "handoffs and transitions." The survey represents pre- and post-comparison. All nurses and hospitalists on both the MICRO and control sides of the study unit had received the TeamStepps curriculum and participated in MICRO rounds by the time of the postintervention survey. We added 3 questions specifically assessing the perception of the efficiency and effectiveness of IDR. Postintervention respondents reflected on their overall impression of IDR, which included their experiences on both sides of the unit, because no group of nurses or hospitalists was exposed only to the MICRO side or the control side of the unit. Responses to survey questions were recorded on a 5-point Likert scale (from "strongly disagree" to "strongly agree" for opinion questions; and "never," rarely," sometimes," "most of the time," and "always" for frequency questions) and given a score from 1 to 5 . The question asking for an overall grade for patient safety was scored from 1 to 5 points corresponding to letter grade choices F, D, C, B, A.

\section{Statistical Analysis}

The sample size was based on the estimate of the baseline rate of the primary outcome of $\mathrm{CD}$ and the projected decrease by the MICRO intervention. A study using the Global Trigger Tool developed by the Institute for Healthcare Improvement provided a best estimate of $16 \%$ as the baseline rate for $\mathrm{CD}$. $^{7}$ A total of 2000 hospitalizations were planned to be included to have a power of at least $80 \%$ to detect a $25 \%$ reduction in the annual incidence of $\mathrm{CD}$ with a 2-tailed type I error rate of 0.05. Comparisons of dichotomous event rates were made using chi square tests at a 2-tailed level for significance of 0.05 . The LOS was analyzed using the nonparametric median test and multivariable regression analysis. We used a generalized linear model with gamma distribution and log link for all analyses of LOS, where LOS was the outcome variable, and intervention vs. control unit type was the predictor variable. Age, sex, race, payer, case mix, and comorbidities defined with the Elixhauser algorithm were used as covariates. ${ }^{8}$ We used multivariable logistic regression for analysis of $\mathrm{CD}$, where the dependent variable was $\mathrm{CD}$. Predictor variables included intervention, patient age, sex, race, payer, case mix and comorbidities. Patient satisfaction data were compared using the chi square test. The Student $t$ test for dependent means was used to analyze the patient safety culture survey data.

The study protocol was submitted to the Icahn Mount Sinai School of Medicine's institutional review board and determined to be exempt from full review.

\section{RESULTS}

A total of 2005 hospitalizations were included over the 12-month study period, consisting of 1089 hospitalizations in the MICRO group and 916 in the control group. Bedside and standard IDR were completed daily, Monday through Friday without exception. The demographic characteristics and comorbidities were similar for the 2 groups (Table). Hospitalizations of patients who were initially admitted to another unit and subsequently transferred to the study unit accounted for $11.1 \%$ of hospitalizations.

Risk-adjusted LOS was similar for the groups (6.6 vs 7.0 days, $P=0.17$, for the MICRO and control groups, respectively). On subgroup analysis, a reduction in LOS was noted for patients transferred to the study unit (10.4 vs 14.0 days, $P=0.02$, for the MICRO and control groups, respectively). The LOS was unchanged for patients admitted directly to the study unit ( 6.0 vs 5.8 days, $P=0.93$ ). There was no difference in the incidence of clinical deterioration for the MICRO or control groups ( $7.7 \%$ vs $9.3 \%$, odds ratio, 0.89 ; 95\% confidence interval, $0.61-1.22, P=0.46$ ).

The finding of a LOS benefit for the MICRO group limited to patients transferred to the study unit prompted a comparison of patients transferred to the study unit and patients directly admitted to the study unit from the emergency department (Appendix 2). Compared to patients admitted directly to the study unit, patients transferred to the study unit were more likely to have Medicaid or no insurance, more likely to be discharged to a facility, had longer LOS, and were more likely to experience CD.

\section{Patient Satisfaction}

There were 175 and 140 responses to the HCAHPS survey for the MICRO and the control groups, respectively. Patients in the MICRO group were more likely to report that "doctors, nurses, or other hospital staff talk with you about whether you would have the help you needed when you left the hospital" ( $88 \%$ vs $78 \%, P=0.01$ ). Responses for all other HCAHPS items were similar for the 2 groups.

\section{Clinician/Staff Survey}

The response rate was 96\% (30 nurses and 17 hospitalists) pre-intervention and 100\% (30 nurses and 22 hospitalists) postintervention. Hospitalists and nurses gave significantly higher scores for the dimensions "teamwork within units," "overall perception of patient safety," and "patient safety grade" on the postintervention survey compared to the pre-intervention survey (Figure 1). Hospitalists and nurses rated the efficiency of IDR and the ability of IDR to identify safety issues higher on the postintervention survey compared to the pre-intervention survey (Figure 2).

\section{DISCUSSION}

We transformed daily IDR from a standard conference room model to a structured bedside model with scripted roles, and performed a rigorous comparison using patient-level data. Our finding that transforming daily IDR from a standard con- 


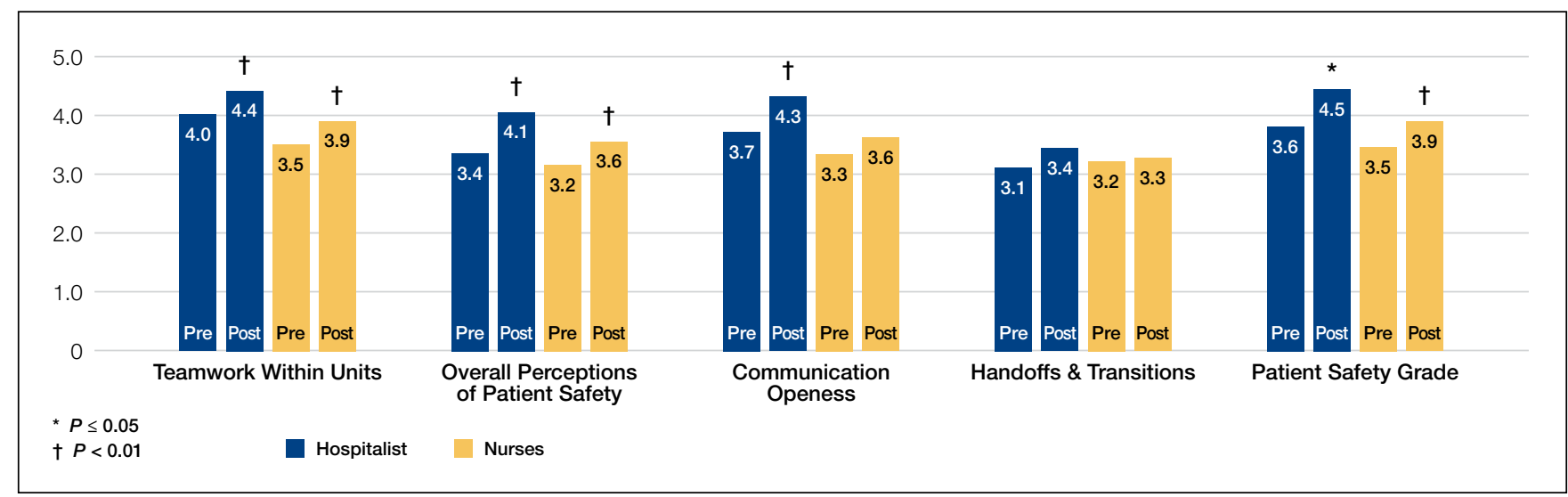

FIG. 1. Patient safety culture dimensions.

NOTE: Abbreviations: pre, pre-intervention survey; post, postintervention survey.

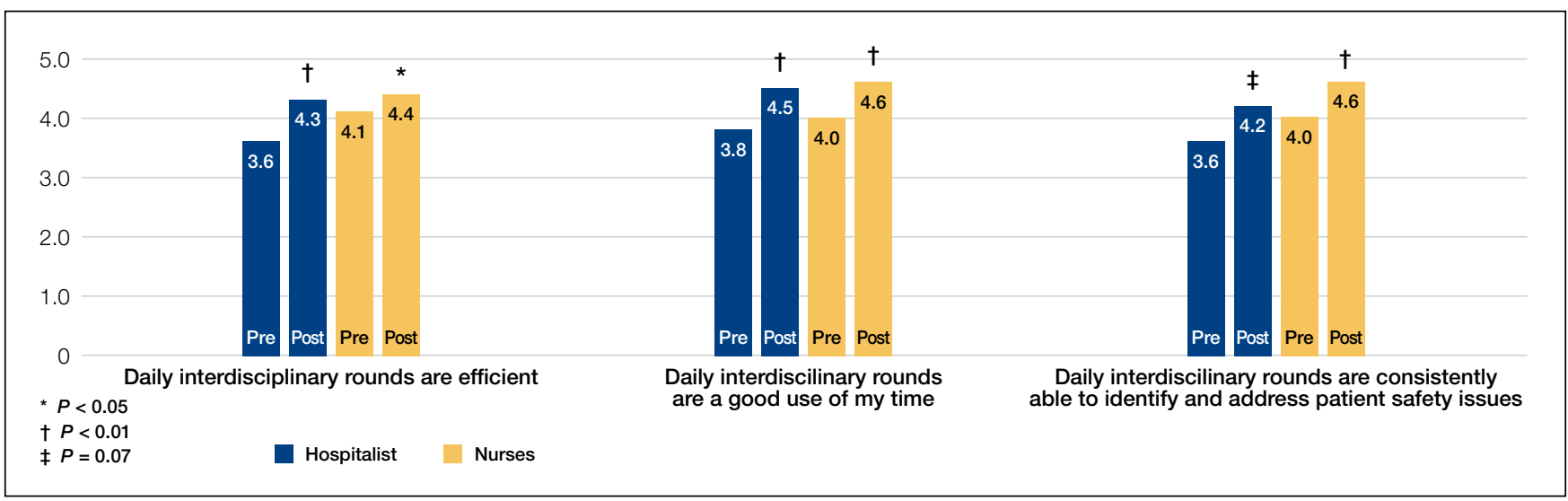

FIG. 2. Hospitalist and nurse perceptions of interdisciplinary rounds.

NOTE: Abbreviations: pre, pre-intervention survey; post, postintervention survey.

ference room model to a bedside model did not significantly reduce LOS suggests either that the model is ineffective or needs to be incorporated into more comprehensive efforts to improve clinical outcomes. Studies suggest that bedside rounding can improve outcomes when implemented in the context of comprehensive restructuring of patient care., ${ }^{4,9}$ Stein et al. ${ }^{4}$ have described the reorganization of a medical ward as an "accountable care unit." The ACU model included daily IDRs at the bedside, as well as geographic-based teams, co-leadership by a hospitalist and nurse manager, and unit-level reporting. Although no definitive conclusions can be drawn based on their descriptive report, transformation of the unit was associated with reduced LOS and mortality. Similarly, Kara et al. ${ }^{9}$ found that the number of elements of an "accountable care team" model implemented by each unit was associated with greater reductions in LOS and cost. In contrast, our findings of a lack of an effect are consistent with a recent cluster-randomized trial by O'Leary et $\mathrm{al},{ }^{10}$ which found that implementation of patient-centered bedside rounds did not improve patient satisfaction or perceptions of shared decision-making compared to units using a model of structured IDRs in a conference room setting.
It is notable that the control groups in both the O'Leary trial $^{10}$ and this study did not represent usual care, because these groups featured localization of the clinical teams and high-quality IDR. In our trial, it is plausible that the control side of the unit was functioning at a high level, which would have decreased our ability to further improve outcomes. Whether restructuring unit processes, including implementation of bedside IDR, improves care compared to usual care without these processes is unknown.

We found that the MICRO intervention significantly decreased LOS compared to the control group for patients transferred to the study unit. This analysis was exploratory and the finding was unexpected. Patients were transferred to the study unit from units of higher acuity, and were more likely to have Medicaid or no insurance and be discharged to facilities rather than home, suggesting that these patients had substantial disposition challenges. It is plausible that this is the population for which bedside IDRs may have the greatest impact. This was a secondary analysis, however, and should be considered as hypothesis-generating for future investigations.

Although the impact on outcomes of bedside IDRs is un- 
TABLE. Demographics and Patient Characteristics

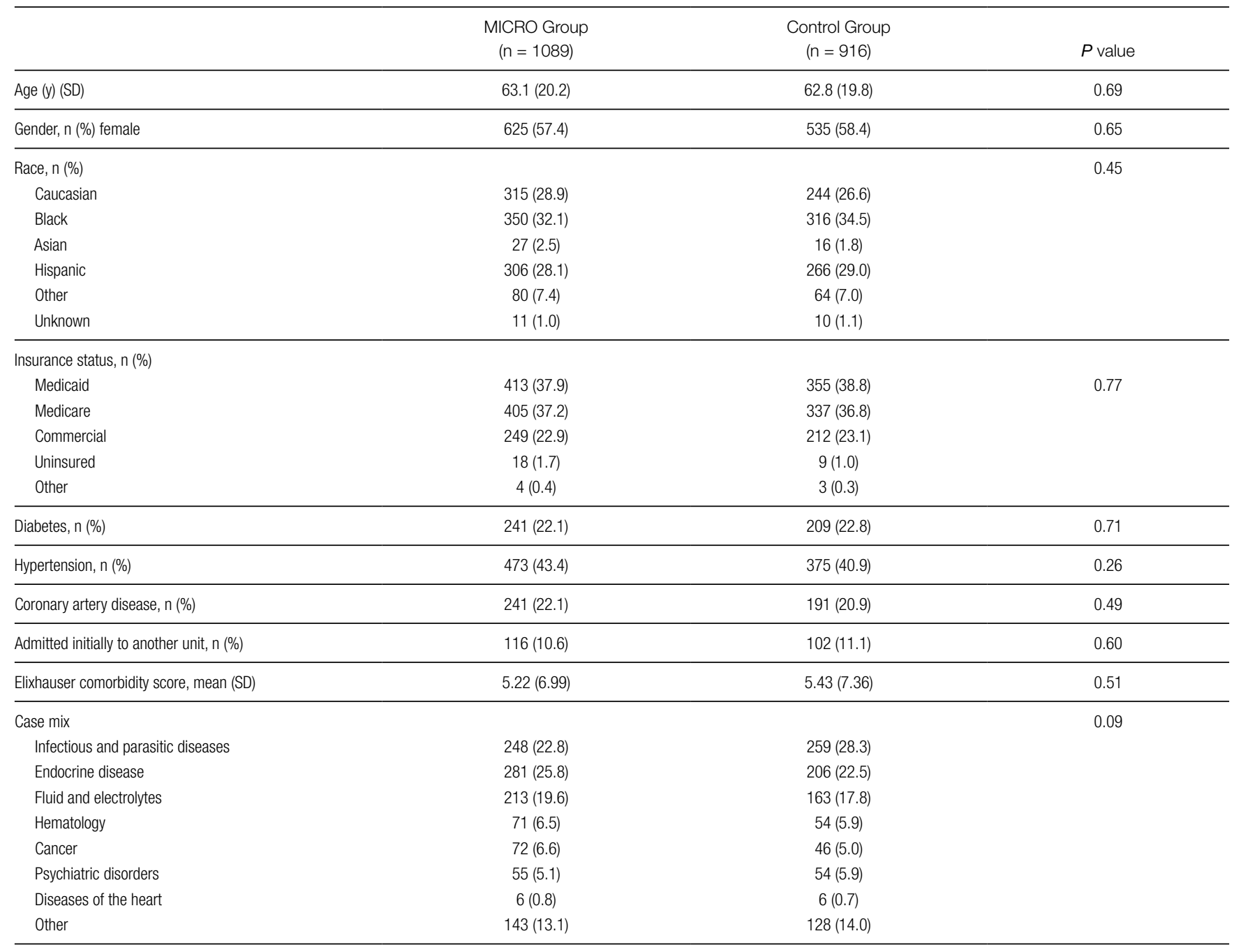

NOTE: Abbreviation: SD, standard deviation.

certain, potential benefits and practical barriers have been examined. Gonzalo et al. ${ }^{11}$ surveyed inpatient physicians and nurses at a hospital employing bedside IDRs and found that the benefits ranked the highest were communication, coordination, and teamwork, and the lowest-ranked benefits were related to efficiency and outcomes. The 6 greatest barriers concerned the time required to complete bedside IDR. These results indicate that the time investiture by staff may be a barrier to widespread adoption. More modest changes, such as increasing the structure of standard conference room rounds, may improve care, although the data are mixed. O'Leary et al. ${ }^{12}$ assessed the value of a structured approach in a conference room setting, which primarily entailed implementing a checklist for newly admitted patients, and found no difference in LOS. Follow-up studies by these investigators found mixed results on the ability of structured IDR to decrease the incidence of adverse events. ${ }^{13,14}$

The results of our AHRQ survey of patient safety culture found that several important aspects of teamwork and safety were perceived as improved by the intervention, including the "overall grade on patient safety." Other studies have similarly shown increases in teamwork and safety ratings through redesign of IDR. O'Leary et al. ${ }^{12}$ surveyed residents and nurses on a unit that implemented a structured, conference room-based IDR and found that providers on the intervention unit rated the teamwork climate higher than providers on the control unit. Our finding that hospitalists and nurses gave higher ratings for IDR being "efficient" and "a good use of my time" on the postintervention survey than the pre-intervention survey suggests that initial concerns about the additional time commitment may be offset by gains in overall efficiency and in development of an environment of enhanced communication, teamwork, and safety.

This study has several limitations. First, the trial may have been underpowered to find small differences between the groups. The trends for decreased LOS and clinical deterioration in the MICRO group may suggest that bedside IDR can provide a small but clinically significant benefit that would 
be statistically significant only in a larger trial. Second, patients were not randomized to the 2 groups. The impact is diminished, however, because the routine hospital process for assigning patients to the 2 areas in which the groups were located is random and based solely on bed availability. Third, nurses and hospitalists caring for patients in the control group likely experienced improved communication practices from the unit-wide TeamStepps education and from participating in the MICRO protocol when caring for patients on the intervention side of the unit. Fourth, we did not collect data on the effectiveness of communication and are unable to assess the fidelity with which the structured protocol was followed or whether interprofessional communication was fostered or hindered. Lastly, the study was implemented on a nonteaching unit at a single academic medical center. The protocol and the results may not be generalizable to other hospitals or units that include house staff.

In conclusion, transforming IDR from a conference room model to a bedside model did not reduce overall LOS or clinical deterioration on a unit using features of an ACU structure. Although several beneficial effects were noted, including a reduction in LOS for patients transferred to the study unit and higher ratings of the patient safety climate and efficiency of IDR, implementing bedside IDR in this setting has marginal benefit. Future studies should assess whether a comprehensive transformation of the inpatient model of care, including patient-centered bedside IDR, geographic cohorting of teams, and co-leadership, improves outcomes compared to models without these features.

Disclosures: This trial was funded by Medline's Prevention Above All Discoveries Grant Program. The authors report no financial conflicts of interest.

\section{References}

1. O'Leary KJ, Sehgal NL, Terrell G, Williams MV; High Performance Teams and the Hospital of the Future Project Team. Interdisciplinary teamwork in hospitals: A review and practical recommendations for improvement. J Hosp Med. 2011;7(1):48-54

2. Gonzalo JD, Wolpaw DR, Lehman E, Chuang CH. Patient-centered interprofessional collaborative care: factors associated with bedside interprofessional rounds. J Gen Intern Med. 2014;29(7):1040-1047.

3. Pannick S, Davis R, Ashrafian H, et al. Effects of interdisciplinary team care interventions on general medical wards. A systematic review. JAMA Intern Med. 2015;175(8):1288-1298.

4. Stein J, Payne C, Methvin A, et al. Reorganizing a hospital ward as an accountable care unit. J Hosp Med. 2015;10(1):36-40.

5. Mohr J, Batalden P, Barach P. Integrating patient safety into the clinical microsystem. Qual Saf Health Care. 2004;13(suppl 2):ii34-ii38.

6. Nelson EC, Batalden PB, Huber TP, et al. Microsystems in health care: Part 1. Learning from high-performing front-line clinical units. Jt Comm J Qual Improv. 2002;28:472-493

7. Rutberg H, Borgstedt Risberg MB, Sjödahl R, Nordqvist P, Valter L, Nilsson L. Characterisations of adverse events detected in a university hospital: a 4-year study using the Global Trigger Tool method. BMJ Open. 2014;4(5):e004879.

8. Elixhauser A, Steiner C, Harris DR, Coffey RM. Comorbidity measures for use with administrative data. Med Care. 1998;36(1):8-27.

9. Kara A, Johnson CS, Nicley A, Niemeier MR, Hui SL. Redesigning accountable care: testing the effectiveness of an accountable care team model. J Hosp Med. 2015;10(12):773-779

10. O'Leary KJ, Killarney A, Hansen LO, Jones S, Malladi M, Marks K, et al. Effect of patient-centred bedside rounds on hospitalised patients' decision control, activation and satisfaction with care. BMJ Qual Saf. 2016;25(12):921-928.

11. Gonzalo JD, Kuperman E, Lehman E, Haidet P. Bedside interprofessional rounds: perceptions of benefits and barriers by internal medicine nursing staff, attending physicians, and housestaff physicians. J Hosp Med. 2014;9(10):646-651.

12. O'Leary KJ, Wayne DB, Haviley C, Slade ME, Lee J, Williams MV. Improving teamwork: impact of structured interdisciplinary rounds on a medical teaching unit. J Gen Intern Med. 2010;25:826-832.

13. O'Leary KJ, Buck R, Fligiel HM, et al. Structured interdisciplinary rounds in a medical teaching unit: improving patient safety. Arch Intern Med. 2011;171(7): 678-684.

14. O'Leary KJ, Creden AJ, Slade ME, et al. Implementation of unit-based interventions to improve teamwork and patient safety on a medical service. Am J Med Qual. 2015;30(5):409-416. 